\title{
Pediatric radiological diagnostic procedures in cases of suspected child abuse
}

\author{
C. Erfurt ${ }^{1}$, G. Hahn², D. Roesner ${ }^{3}$, U. Schmidt ${ }^{1}$ \\ ${ }^{1}$ Institute for Legal Medicine, Technical University Dresden, Dresden, Germany; Christine.Erfurt@tu-dresden.de \\ ${ }^{2}$ Institute and Polyclinic for Radiological Diagnostics, Pediatric Radiology Department, University Hospital “Carl Gustav Carus”, \\ Dresden, Germany \\ ${ }^{3}$ Clinic and Polyclinic for Pediatric Surgery, University Hospital “Carl Gustav Carus”, Dresden, Germany
}

Received 10 November 2009; revised 23 December 2009; accepted 25 December 2009.

\begin{abstract}
Advanced and specialized radiological diagnostic procedures are essential in cases of clinically diagnosed injuries to the head, thorax, abdomen or extremities of a child, especially if there is no case history or if the reporting of an inadequate trauma suggests battered child syndrome. In particular, these diagnostic procedures should aim at detecting lesions of the central nervous system (CNS), so that the treatment can be immediately initiated. If the diagnostic imaging reveals findings typically associated with child abuse, accurate documentation constituting evidence, which will stand up in court, is required to prevent any further endangerment of the child's welfare.
\end{abstract}

Keywords: Child Abuse; Battered Child Syndrome; Shaken Baby Syndrome; Non-Accidental Injury;

Pediatric Radiological Diagnostics

\section{INTRODUCTION}

Children have the right to a non-violent childhood and adolescence. This right is enshrined in the UN Convention on the Rights of the Child, which was adopted by the United Nations General Assembly in November 1989. Among the fundamental children's rights are the right to a non-violent upbringing (in Germany Section 1631 Subsection 2 of the Civil Code) and the right to protection from physical, emotional or sexual abuse.

The statistics on crime published by the Federal Criminal Police Office in Germany show that even today these rights are not secured for all children and adolescents [1].

In 2006, a total of 597, 504 cases of physical injury (code number 2200) were reported, including 3,640 cases of child abuse (code number 2231). Boys were the vic- tims in $55.2 \%$ of cases, and in 12 cases the physical injury resulted in death.

The number of cases of sexual abuse against children (code number 1310) was much higher, with a total of 15,185 victims in 12,765 registered cases.

These data are consistent with our own daily experience, in which we have observed over the past few years a major increase in the number of children examined due to suspected physical or sexual abuse.

\section{FORMS OF ABUSE}

The term child abuse is very hard to define [2]. The expression threat to child well-being does not comprise accidental, intentional or unintentional, violent, mental or physical damage to a child leading to injuries, abnormal development or even death and affecting or threatening the well-being and the rights of a child.

The following forms of violence can be distinguished [3]:

\subsection{Physical Abuse}

Physical abuse occurs when violent behavior on the part of the parents or other caregivers causes physical injury to the child. Physical injuries should be considered abuse if not resulting from an accident; if the type of injury is not consistent with the alleged cause of the injury or explanation of how the injury occurred; if there are reasonable grounds for suspicion or an admission has been made that the injury was caused by a person exercising parental responsibility for the child, responsible for the care of the child, or an attachment figure for the child; or if such a person purposely did not prevent the injury to the child. Physical abuse also encompasses forms of injury such as attempted drowning, suffocation or the administration of damaging substances.

\subsection{Emotional Abuse}

Emotional abuse is defined as a hostile or neglectful 
attitude, the rejection or ignoring of the child by the parents or parental figures-a behavior that seriously damages the child's sense of personal identity and selfesteem. Also included is emotional neglect, when the parents or caregivers fail through negligence to provide the child with the family atmosphere required for healthy emotional development (for example through constant emotional coldness or ignoring the child, as silent forms of child abuse).

\subsection{Physical Neglect}

Physical neglect means that the parents or other caregivers fail completely or partially to provide for the child's survival or well-being, i.e. health care, nourishment, clothing, health promotion, protection and supervision.

\subsection{Sexual Abuse}

Sexual abuse is the involvement of children and adolescents in all types of sexual activity and/or sexual exploitation by adult reference persons to whom the child cannot give informed consent owing to ignorance, dependence, developmental immaturity or fear.

Child pornography is a form of sexual abuse, as is also the confrontation of minors with representations of sexuality in a manner that is inappropriate to the child's stage of development. In every case, responsibility for such mistreatment is borne by the perpetrator. Incest is a special form known as 'intrafamilial child sexual abuse', committed by a member of the family group. Child sexual abuse also refers to actions performed with the intention of sexually stimulating a child or using a child for the purpose of sexual arousal, either of the perpetrator or of another person.

Children in particular are often unable to speak outeither because they are insufficiently developed as infants or toddlers, or as a result of fear. A thorough physical examination in cases of suspected mistreatment or sexual abuse is an important milestone in both the diagnosis and 'therapy' of the abuse. The detection and ascertainment of traumatic findings and their attribution is an important diagnostic criterion in cases of suspected child abuse.

Infants and toddlers are particularly challenging in this context. In instances of suspected physical abuse, the examination should not be restricted solely to the external inspection of the body, since osseous injuries are frequently not detected through clinical examination. A full skeletal survey using radiological imaging techniques can detect or rule out bone lesions resulting from traumatic injury, therefore constituting an essential component of the investigations in suspected child abuse cases.

Both action and failure to provide care can constitute a threat to a child.

The following text briefly deals with the possible consequences of physical violence. Based on the daily experiences made by the Doctor of Legal Medicine and the Pediatric Radiologist it can be said that any form of physical violence one can think of can also occur when a child is abused. Typical forms of violence are punches, kicks, pushes against the wall and the use of objects (sticks, etc.).

Consequently, the affected children suffer from injuries such as hematomas, swellings of soft parts or injuries to the soft parts and skin (contused wounds or cuts). Additionally, there can be hidden injuries, such as damaged organs and fractures.

A special form of physical abuse is the shaken baby syndrome. The term "Whiplash shaken baby syndrome" goes back to Caffey [4]. Severe shaking of a baby leads to strong shearing forces due to acceleration and deceleration. Babies and toddlers are not able to hold their head, which is very heavy compared with their body, when they are shaken severely. As a result there will be cranial hemorrhages (subdural and subarachnoid hemorrhages), hemorrhages in the retina and lesions of cranial tissue [5]. The shaken baby syndrome can lead to death. The affecting forces have been determined extensively in the finite element analysis [6]. The scope of injuries resulting from shaken baby syndrome comprises not only intracranial injuries, but also frequently injuries of the skeletal system in particular rib fractures and finger marks [7]. Based on literature one can assume that shaken babies show clinically diagnosable symptoms [8].

The strategy of examination depends on the clinical situation and the age of the child.

Besides the clinical examination, radiological imaging examination methods are to be applied.

\section{IMAGING PROCEDURES}

Accordingly, we aim to presently discuss the various uses of radiological diagnostic imaging in this context, including an assessment of their respective value and the necessary indications.

In instances of clinical suspicion of child abuse, the following imaging procedures may be used: skeletal radiography, computer tomography (CT) and/or, preferably, magnetic resonance imaging (MRI) investigations and sonography $[9,10]$.

\subsection{Full Radiographic Skeletal Survey}

A full radiographic skeletal survey should always be performed in children up to 2 to 3 years old if child abuse is suspected [11]. All the extremities, the thorax and pelvis should be x-rayed from one viewing angle and the cranium, spine and fracture areas should be $\mathrm{x}$-rayed from two viewing angles (Figure 1). The radiographic images of the extremities should include the 


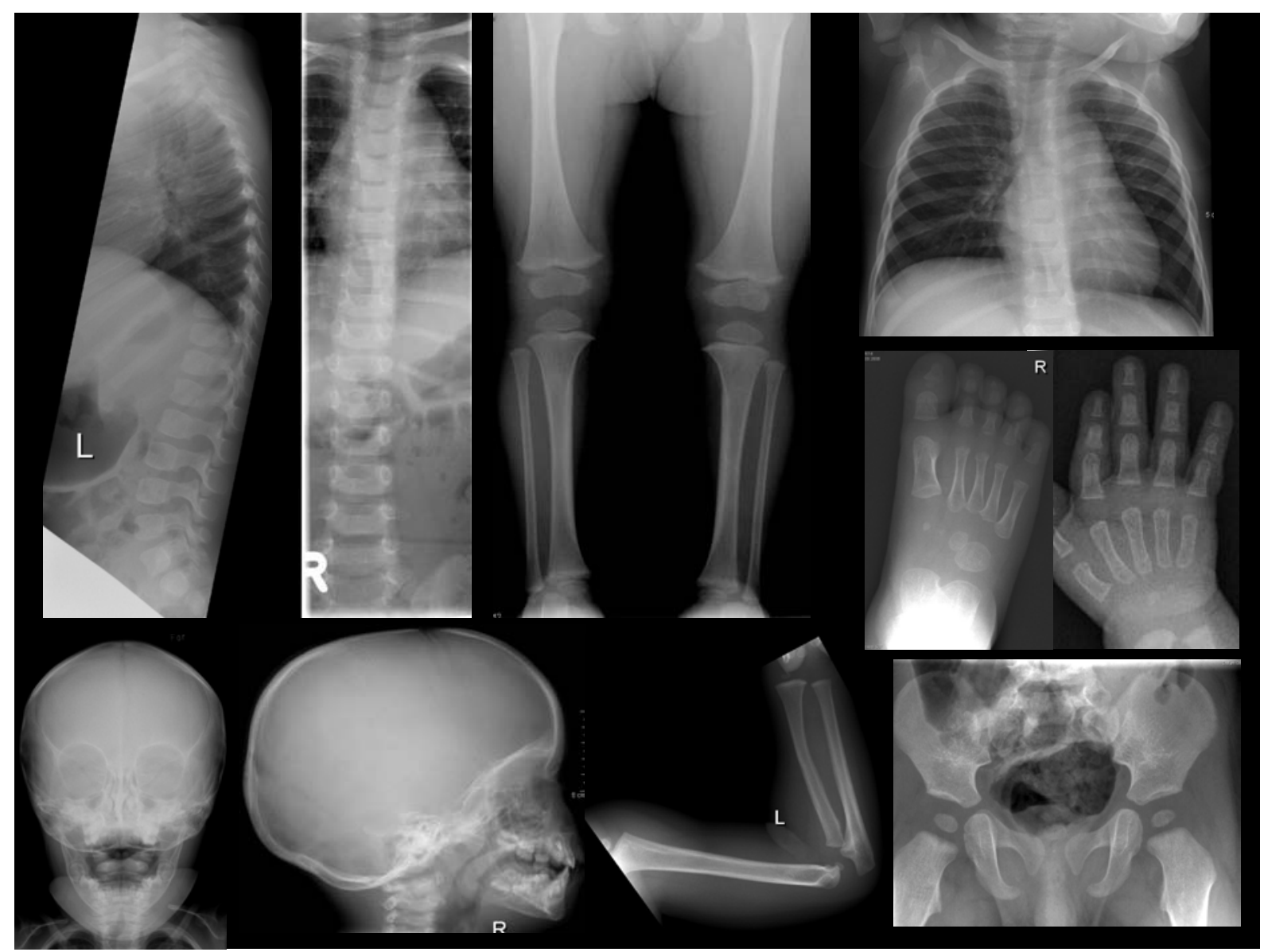

Figure 1. Skeletal radiographs in an instance of suspected child abuse in a child under the age of 2-3 years.

hands and feet, or these should be x-rayed selectively. The procedure known as a 'babygram', i.e. producing a single radiographic image of the whole child, is not suitable for the purpose of confirming or ruling out child abuse, because the metaphyses, in particular, cannot be adequately assessed. In older children, selected skeletal areas should be $\mathrm{x}$-rayed from two viewing angles if there is clinical suspicion of trauma-induced lesions (hematomas, palpable callus formation, axial deviation).

\subsection{Sonography}

It depends on the clinical situation whether an ultrasound examination is advisable. Sonography is of utmost importance in case of suspected organic injuries to the abdomen and the soft parts of the extremities.

Sonographic examination of the skull can be used in infants with an open fontanelle. However, the value of such examination must be critically assessed. Non-detection of objective findings in the sonogram (fresh and older areas of bleeding) does not necessarily rule out the existence of such lesions, as shown by some cases in the investigation material examined at the Dresden Institute of Legal Medicine.

\subsection{Computer Tomography}

Computer tomography of the skull is often the first imaging procedure used for the investigation of injuries to the skull and brain.

In cases of blunt trauma to the abdomen, ultrasound should be the first imaging procedure used for detecting free abdominal fluid or injuries to the parenchymatous organs in the upper abdomen and kidneys. In instances of suspected child abuse, the subsequent performance of a computer tomography (using a contrast medium) on the abdomen is the gold standard. Serious thoracic traumas can also be investigated further by means of computer tomography.

\subsection{Magnetic Resonance Imaging}

Magnetic resonance imaging is primarily used when investigating intracranial or spinal injuries.

\subsection{Skeletal Scintigraphy}

Skeletal scintigraphy has now practically ceased to be used in Germany in cases of suspected child abuse, because this procedure is unable to provide relevant an- 
swers in the context of child abuse, since epiphyseal and metaphyseal fractures in the zones characterized by physiological activity (growth areas of the epiphyses) cannot be detected, and the age of existing fractures cannot be determined by this method. Furthermore, the child would be subjected to high radiation exposure despite the limited usefulness of the procedure. Only in cases of occult rib fractures can skeletal scintigraphy be helpful [10].

\section{ANATOMY OF THE BONES DURING CHILDHOOD}

The skeletal anatomy of a child is considerably different from that of an adult. On a conventional radiograph only the ossified parts are visible, while the cartilaginous areas of the growth zones do not appear on a radiographic image. The zones on the long bones which are relevant for height growth are the epiphysis, epiphyseal plates and the metaphysis (Figure 2).

Typical childhood fractures are greenstick fractures, in which the cortex is not completely ruptured due to the high degree of elasticity of the bone.

\section{SKELETAL TRAUMA}

A fracture can be the result of various forms of violence. Its localization and the age of the fracture are essential indicators for its assessment. Skeletal traumatic lesions are not usually life-threatening injuries, but often a definitive indicator of child maltreatment [12]. The radiographic skeletal images should meet the very highest quality standards (spatial resolution: 10 line pairs per $\mathrm{mm}$, without grid) and should be performed by experienced personnel under the supervision of an experienced pediatric radiologist, when available.

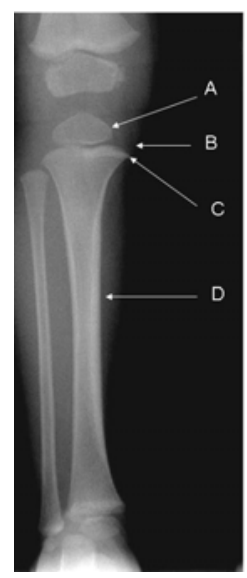

Figure 2. Anatomy of a child's bones in a radiograph, as exemplified by the lower leg with the knee joint (A-ossification nucleus of the epiphysis, Bepiphyseal plate, C-metaphysis, D-diaphysis).

\subsection{Determining the Age of a Fracture}

A question often posed both in criminal and in family law proceedings pertains to the age of fractures or the possible occurrence of the traumatic injuries at different times. When determining the age of fractures, particularly when multiple fractures are detected, the following parameters have to be taken into account:

1) 0-10 days: soft tissue swelling, edemas, bleeding, fracture lines and fragments (Figure 3);

2) 7-10 days: earliest callus formation;

3) 10-14 days: clearly evident callus formation;

4) up to 8 weeks: periosteal new bone formation;

5) subsequent resorption: after 3 months the fracture may have healed without residue.

Skull fractures do not result in callus formation, so that the age of a fracture cannot be determined by means of an x-ray image alone. The approximate age of the injury (fresh or older) can be determined, however, by reference to soft tissue trauma, such as swelling or hemorrhaging.

\subsection{Types of Fracture}

\subsubsection{Metaphyseal and Epiphyseal Fractures}

Metaphyseal and epiphyseal fractures have a high specificity for child abuse [13].

Multiple subphyseal micro-fractures at the connection between the metaphysis and the epiphyseal plate are usually attributable to centrifugal and rotational forces. Metaphyseal corner/chip fractures, bucket-handle fractures and metaphyseal fractures are indicators of maltreatment via this mechanism and are virtually proofpositive when summing up all assessments (Figure 3). The readaptation of metaphyseal corner fractures generally takes place within approximately 18 days [14].

\subsubsection{Diaphyseal Fractures}

Diaphyseal fractures have a low specificity for child abuse, since they also occur frequently in accidental traumas. However, they occur significantly more frequently than metaphyseal fractures [15].

Humerus fractures are suspicious signs of non-accidental injury, particularly in children under the age of 15 months (Figure 4).

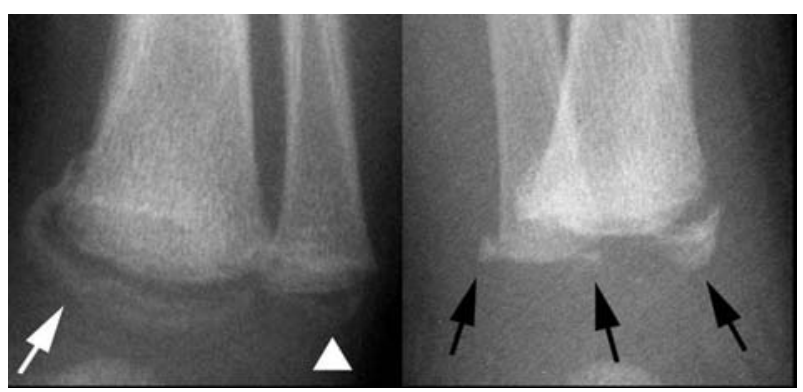

Figure 3. Radiograph of the distal lower leg with metaphyseal fractures evident as bucket-handle fracture and corner fractures. 
Lower arm fractures in the middle of the shaft can be evaluated as self-defense injuries (parry fractures) resulting from an impact effect (Figure 5).

Femoral fractures during the first year of life are suspicious as indicators of external force having been applied.

Tibial fractures, distal metaphyseal and epiphyseal, and proximal metaphyseal, may occur through child abuse.

Fractures of the sternum, scapula and pelvis are observed very rarely in cases of child abuse, as the application of great force is required to cause them.

Spinal fractures are also the result of massive traumas and only occur very rarely as compression fractures in instances of child abuse.

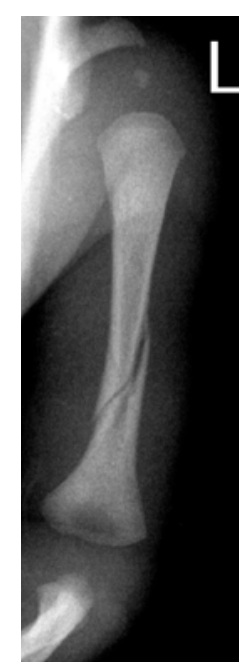

Figure 4. Radiograph of the left upper arm: spiral fracture of the humeral shaft in an infant.

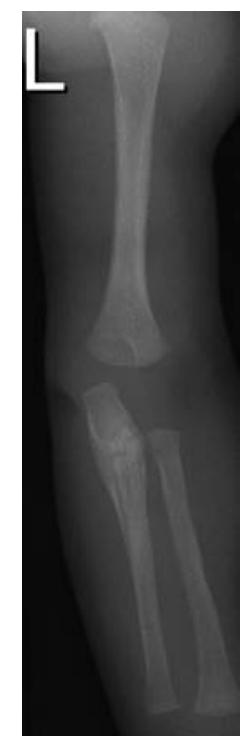

Figure 5. Radiograph of the left arm: proximal fracture of the ulna, healed with callus formation.

\subsubsection{Periosteal Reactions}

Rotational and flexion forces, in particular, can result in the detachment of the periosteum, which is elastic in the diaphysis but stiff in the metaphysis. The sub-periosteal hemorrhaging thus caused leads to the development of areas of periosteal ossification, which later become visible (Figure 6).

\subsubsection{Rib Fractures}

Rib fractures and particularly serial rib fractures have a high specificity for the detection of child abuse. When thorax compression has taken place in cases of shaken baby syndrome, serial rib fractures are often observed [16]. These are found in both the posterior paravertebral and in the lateral regions. These fractures only become visible on a radiograph after the start of callus formation after approximately 7 days (Figure 7).

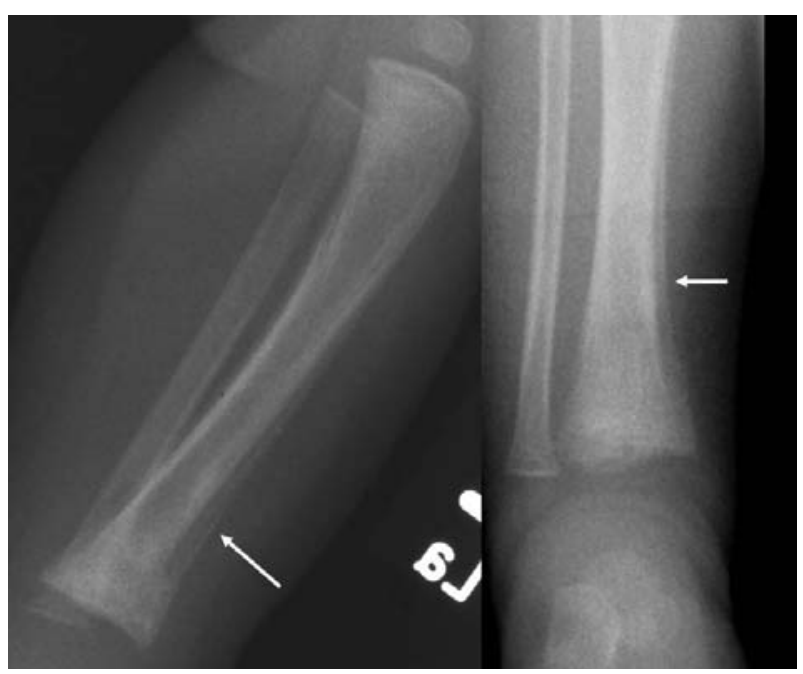

Figure 6. Radiograph of the left lower leg from two viewing angles: healing metaphyseal fracture on the distal tibia with periosteal calcification.

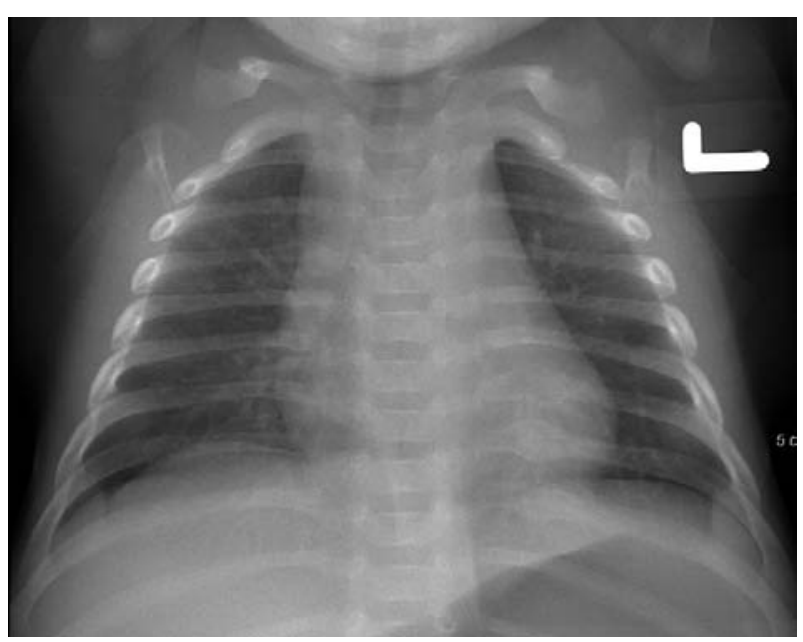

Figure 7. Radiograph of the thorax: left paravertebral region of ribs 7-9 showing post-fracture callus formation. 


\subsection{Differential Diagnoses of Skeletal Injuries Following Child Abuse}

The evaluation of ossified lesions always requires important differential diagnoses to be considered [10] and should therefore be conducted by an interdisciplinary team.

Significant differential diagnoses are:

1) accidental traumas

2) birth trauma (clavicle, humerus)

3) premature infant osteopenia (Figure 8)

4) metabolic disorders (rickets, Menkes syndrome etc.)

5) medication (Methrotrexate, Prostaglandin E etc.)

6) infections (syphilis, osteomyelitis etc.)

7) neuromuscular disorders (infantile cerebral palsy)

8) neoplasias (leukemia, histiocytosis X, metastases)

9) infantile cortical hyperostosis

10) osteogenesis imperfecta

\subsection{Cranial Trauma/Shaken Baby Syndrome}

Indicators of child abuse are calvarial fractures (Figures 9 and 10) and sutural diastasis, with the ping-pong ball fracture of the soft calvarium of the young infant and the growing skull fracture being particularly significant.

In the case of the ping-pong ball fracture, the exertion of external force leads to a permanent inward deformation of the elastic cranial vault of the young infant (Figure 11).

The growing skull fracture is caused by brain tissue and cerebral membranes being trapped in the fracture fissure, preventing the healing of the bone (Figure 12).

In this paper, we do not intend to discuss the clinical characteristics and mechanisms involved in shaken baby syndrome. A detailed presentation on this topic has been published in different journals [17-19].

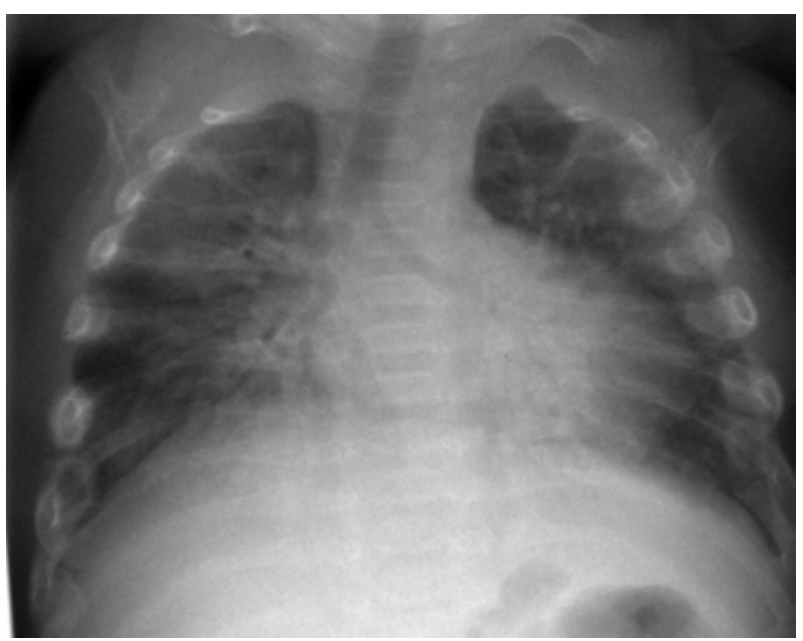

Figure 8. Radiograph of the thorax: premature infant osteopenia and bronchopulmonary dysplasia with condition after multiple rib fractures.

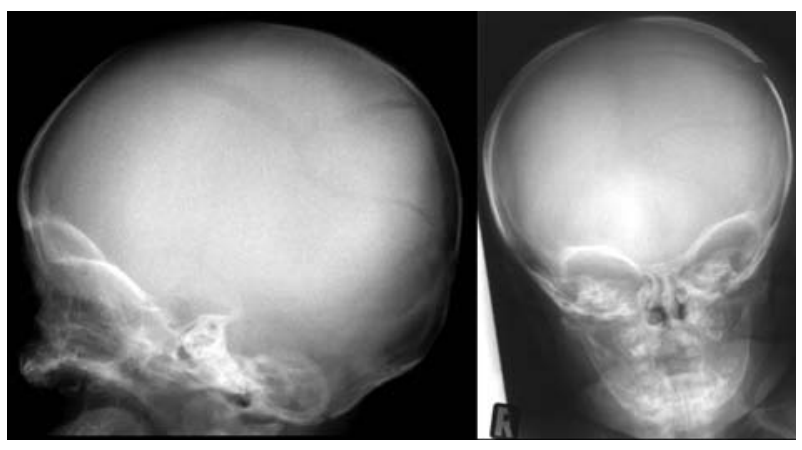

Figure 9. Radiograph of the skull from two viewing angles: cranial burst fracture on the left parietal bone.

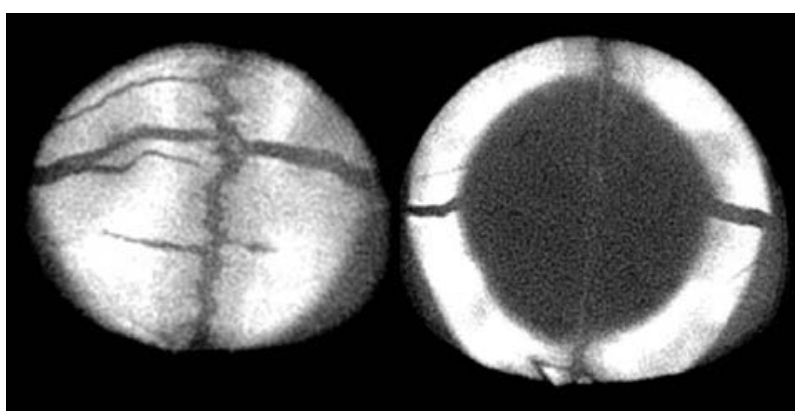

Figure 10. CT image of the skull: biparietal calvarial fracture crossing the sagittal suture.

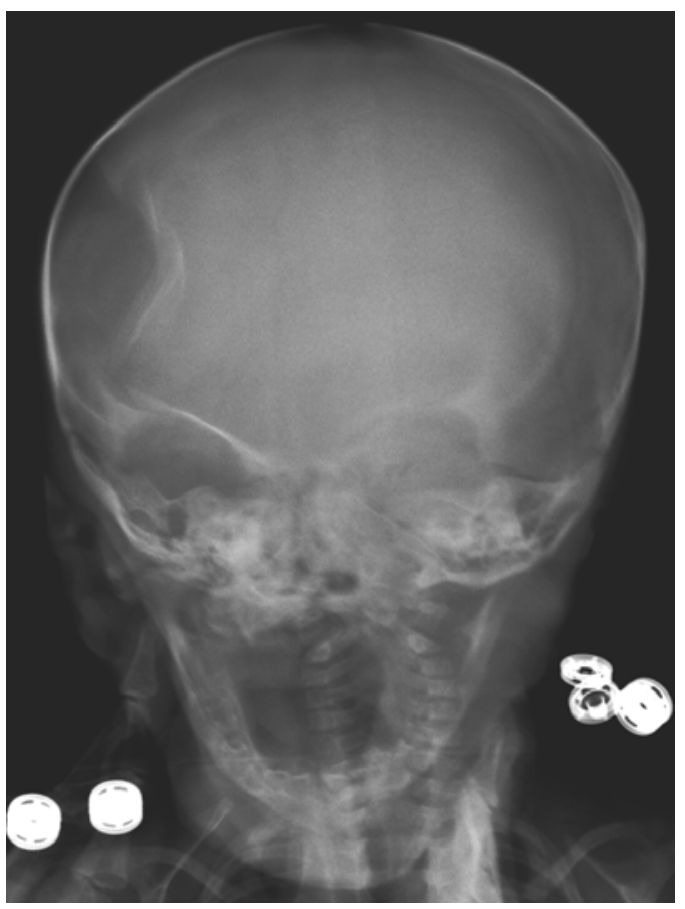

Figure 11. Radiograph of a skull with a ping-pong ball fracture on the right parietal bone.

Intracranial injuries, however, are usually more serious and can be life-threatening [20]. In the case of a child presenting with neurological abnormalities, a cranial 
CT scan is of decisive importance in the highly acute phase owing to its almost unlimited availability and the rapidity in which it can be performed enabling decisions to be made quickly about the diagnosis and the necessary treatment. The CT has a high degree of sensitivity in detecting acute intraparenchymatous, subarachnoid, subdural and epidural hemorrhaging (Figure 13). Pathological changes which may require neurosurgical intervention can be detected with certainty, and critically ill children can be treated quickly. Accompanying cranial and facial fractures can also be diagnosed. Sonography via the open large fontanelle of an infant is an important examination method in the follow-up monitoring of an intracranial injury. MRI is the best imaging method for showing the full extent of the cranial injuries. The detection of extraparenchymatous fluid accumulation, intraparenchymatous hemorrhaging, contusion areas, diffuse axonal injury and cerebral edema requires T1-weighted, T2-weighted, FLAIR, T2-gradient echo and diffusionweighted sequences from several viewing angles (Figure 14). MRI has the highest degree of sensitivity and specificity for the diagnosis of child abuse, and therefore this form of imaging should always be performed in cases of suspected child abuse.

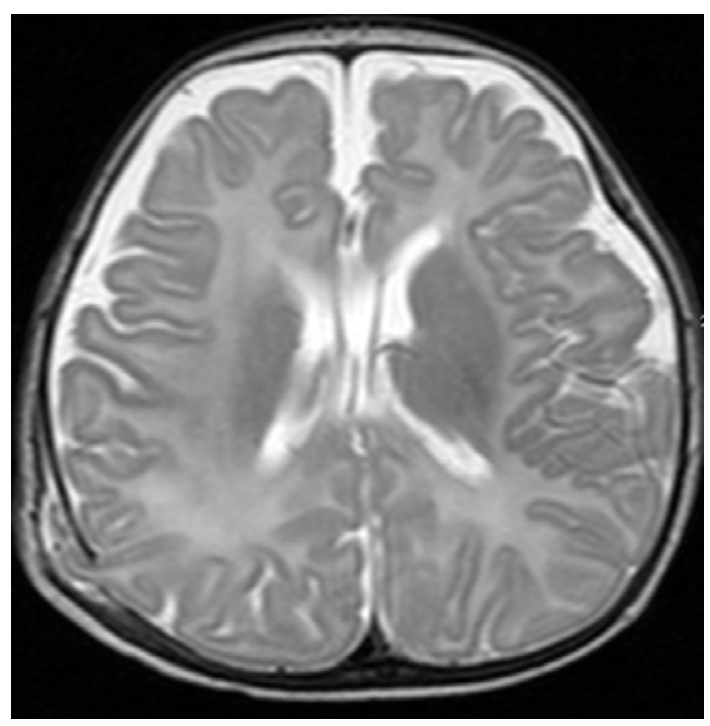

Figure 12. MRI image of a skull: T2-weighted sequence with growing skull fracture on the right parieto-occipital bone and trapped cerebral parenchyma in the subperiosteal area.

A further aspect is the follow-up monitoring in cases of suspected shaken baby syndrome. Due to the serious lesions of the cerebral tissue caused by shaking, these injuries can lead to general cerebral atrophy with dilation of the inner and outer subarachnoid spaces, or to a circumscribed cystic encephalomalacy (Figures 15 and 16).

Bilateral subdural hemorrhaging is generally indicative of child abuse involving shaken baby syndrome.
Subdural hemorrhaging in the supra and infratentorial regions or in the interhemispheric fissure substantiate the suspicion of shaken baby syndrome. Unilateral subdural hemorrhaging may also be caused by a simple fall.

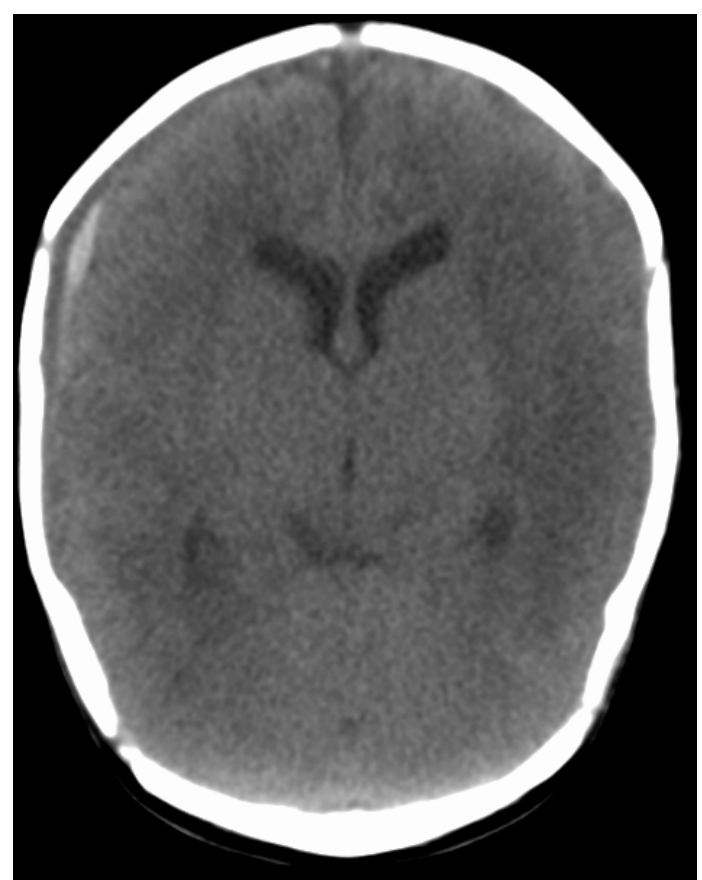

Figure 13. CT image of a skull: subdural hematoma in the right frontotemporal region and focal cerebral edema in the left frontotemporal region.

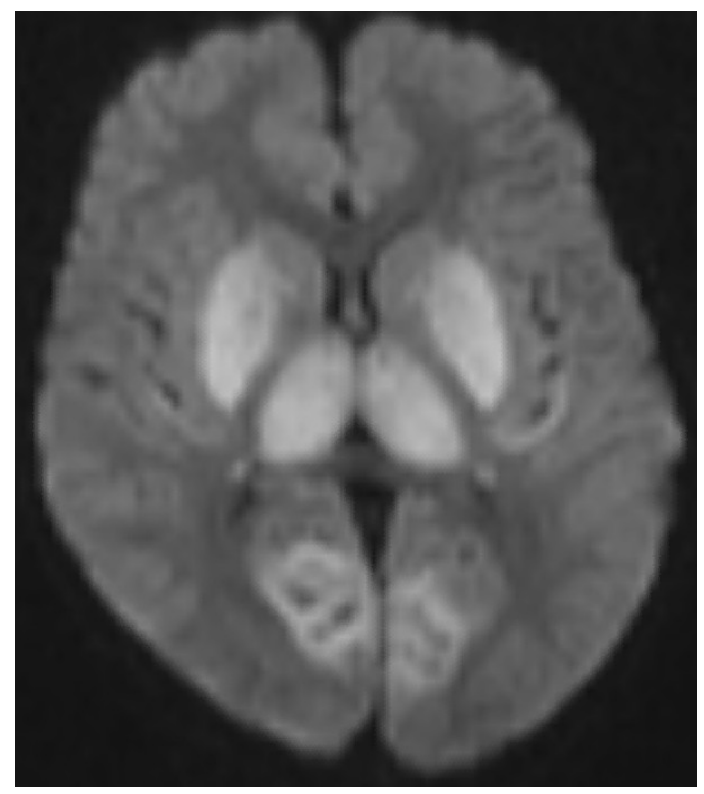

Figure 14. MRI image of a skull: diffusion- weighted sequence with hypoxically damaged basal ganglia and cerebral parenchyma in the occipital region on both sides as an indicator of a highly acute fresh parenchymal lesion. 


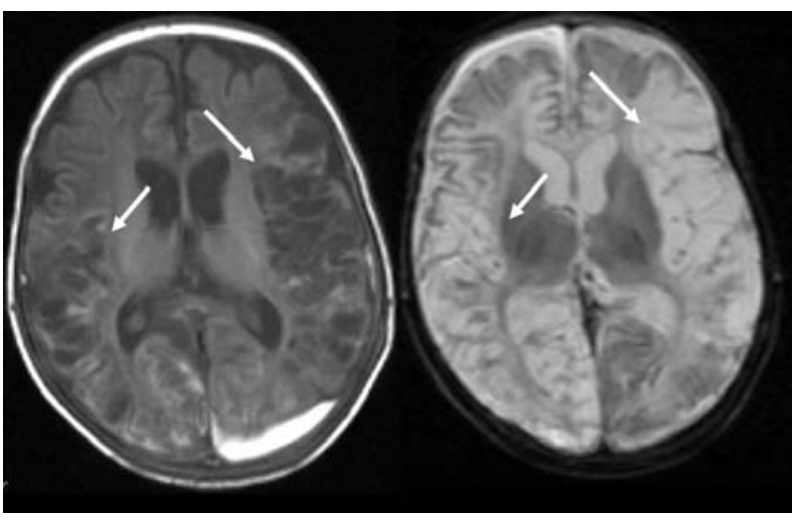

Figure 15. MRI image of a skull: clearly evident bilateral cystic encephalomalacy with a hemorrhage component in the cortical region and a subdural hematoma in the left occipital area extending into the interhemispheric fissure.

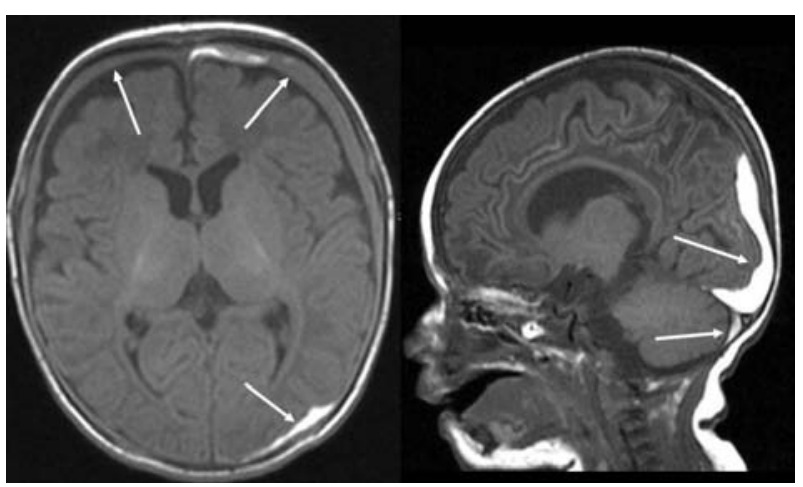

Figure 16. MRI image of a skull: subdural hematomas on both sides in the supra and infratentorial regions with different signal intensity, which may be indicative of the injuries having been sustained at different times.

When assessing cerebral changes using MRI imaging, it is essential that consequences of abuse are distinguished from perinatal brain damage. In premature and newborn infants, specific patterns of brain damage occur, the dynamics of which can be observed in follow-up monitoring and which can therefore usually be distinguished from injuries resulting from child abuse (for assessment purposes, the birth mechanism should be known, if possible). For the purposes of differential diagnosis, vitamin $\mathrm{K}$ deficiency and glutaraciduria must be ruled out.

\subsection{Blunt Trauma to the Thorax and Abdomen}

Serious injuries to the thorax with suspected intrathoracic organ damage can be diagnosed quickly and safely using computer tomography. In cases of suspected child abuse, a blunt abdominal trauma is initially investigated through sonographic examination of the abdomen. If this reveals free abdominal fluid or suspected organic injuries, computer tomography must be used, since it is the most sensitive imaging technique. By this method, ruptures or intramural hematomas in the gastrointestinal tract or tears in the parenchymatous organs can easily be detected through abdominal or retroperitoneal examination. However, serious thoracic or abdominal traumas do not occur frequently in child abuse.

\section{CONCLUSIONS}

The assessment of child abuse requires a multi-professional interdisciplinary approach. In cases of suspected physical abuse, differential diagnosis is necessary taking into account the age of the child and the clinical symptoms. The radiological investigations should comply with the very highest quality standards, so as to be able to produce a diagnosis that will stand up in court while also minimizing the child's exposure to radiation. Final assessment of the images should be left to an experienced pediatric radiologist.

\section{REFERENCES}

[1] Polizeiliche Kriminalstatistik Deutschland 2006 http://www.bka.de/pks/pks2006/download/pks-jb_2006_ bka.pdf (Accessed December 2009).

[2] Stark, M.M. (2005) Clinical forensic medicine a physician's guide. Humana Press.

[3] Erfurt, C., Roesner, D., Scholz, M., Ostwaldt, F. and Schmidt, U. (2006) Gewalt gegen Kinder-Misshandlung Minderjähriger. Ärzteblatt Sachsen, 11, 1-45.

[4] Caffey, J. (1972) On the theory and practice of shaking infants. Its potential residual effects of permanent brain damage and mental retardation. American Journal of Diseases of Children, 124, 161-169.

[5] Maxeiner, H. (2001) Demonstration and interpretation of bridging vein ruptures in cases of infantile subdural bleedings. Journal of Forensic Sciences, 46, 85-93.

[6] Roth, S., Raul, J.-S., Ludes, B., Willinger and Finite R. (2007) Element analysis of impact and shaking inflicted to a child. International Journal of Legal Medicine, 121, 223-228.

[7] Gilliland, M.G. and Folberg, R. (1996) Shaken Babiessome have no impact injuries. Journal of Forensic Sciences, 41, 114-116.

[8] Gilliland, M.G.F. (1998) Interval duration between injury and severe symptoms in nonaccidental head trauma in infants and young children. Journal of Forensic Sciences, 43, 723-725.

[9] Stöver, B. (2001) Kindesmisshandlung. Bildgebende Diagnostik. Radiologie up2date, 1, 259-272.

[10] Stöver, B. (2007) Bildgebende Diagnostik der Kindesmisshandlung. Radiologe, 47, 1037-1049.

[11] German AWMF-Guidelines (AWMF-Leitlinien). Pädiatrische Radiologie Verdacht auf Misshandlung-Bildgebende Diagnostik. http://www.kinder-radiologie.org/; 2004 (Accessed December 2009).

[12] Silverman, F. N. (1953) The roentgen manifestations of 
unrecognized skeletal trauma in infants. ARJ American Journal of Roentgenology, 69, 413-427.

[13] Kempe, C., Silverman, F.N., Steele, B.F. et al. (1962) The battered child syndrome. Journal of the American Medicinal Association, 181, 105-112.

[14] Oppermann, H.C. (2004) Röntgenmorphologische Skelettbefunde beim Battered Child Syndrome (Non Accidental Injury). In: Oehmichen M, Kaatsch HJ, Bosinski HAG (ed.) Gewalt gegen Frauen und Kinder. Bestandsaufnahme-Diagnose-Prävention. Schmidt Römhild, 32, 167179.

[15] Sorantin, E. and Lindbichler, F. (2000) Die nicht unfallbedingte Verletzung (battered child). Radiologe, 42, 210-216.

[16] Herrmann, B. (2008) Nichtakzidentelle Kopfverletzungen und Schütteltrauma. Rechtsmedizin, 18, 9-16.
[17] Caffey, J. (1974) The whiplash shaken infant syndrome: manual shaking by the extremities with whiplash-induced intracranial and intraocular bleedings linked with residual permanent brain damage and mental retardation. Pediatrics, 54, 396-403.

[18] Debertin, A.S. and Sperhake, J.P. (2008) Untersuchung und Dokumentation des nichtakzidentellen SchädelHirn-Traumas im Säuglings-und Kleinkindalter. Rechtsmedizin, 18, 17-22.

[19] Duhaime, A.-Ch., Gennarelli, T.A., hibault, L.E. et al. (1987) The shaken baby syndrome. A clinical, pathological and biomechanical study. Journal of Neurosurgery, 66, 409-415.

[20] Tutsch-Bauer, E., Meyer, H.J. and Monticelli, F. (2005) Schütteltrauma. Rechtsmedizin, 15, 399-408. 